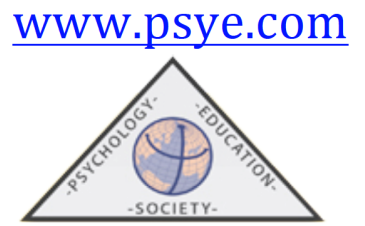

Psychology, Society, \& Education, 2019. Vol. 11(2), pp. 253-266

ISSN 2171-2085 (print) / ISSN 1989-709X (online)

Doi 10.25115/psye.v10i1.2268

\title{
Estigma en los profesionales de la Salud Mental: una revisión sistemática
}

\author{
Noelia NAVARRO GOMEZ y Rubén TRIGUEROS RAMOS
}

\author{
Universidad de Almería, España
}

(Recibido el 18 de Enero de 2018; Aceptado el 12 de Marzo de 2019)

\begin{abstract}
RESUMEN: El efecto de la estigmatización en los profesionales de la salud ha sido un tópico que ha recibido relativamente poca atención. Dado el influyente papel de los profesionales en los procesos de recuperación, es de vital importancia conocer el estado de la cuestión en lo relativo a las creencias y actitudes de los mismos. El objetivo por tanto es conocer el impacto de los procesos estigmatizantes en los profesionales de la salud. Para ello, se plantea una revisión sistemática de los estudios disponibles en la literatura especializada. Los resultados apuntan hacia la existencia de ciertos niveles de estigma entre los profesionales de la salud. Las opiniones de los profesionales hacia los pacientes no difieren significativamente de las de la población general en distancia social. Las actitudes discriminatorias varían en función tanto de las condiciones, como de la patología de los pacientes, siendo la psicosis el trastorno más castigado por el estigma.
\end{abstract}

Palabras clave: Estigma; salud mental; profesionales de la salud; discriminación.

\section{Stigma in Mental Health professionals: a systematic review}

\begin{abstract}
The effect of stigmatization on health professionals has been a topic that has received relatively little attention. Due to the influential role of professionals in recovery processes, it is vital to know the status of the issue in relation to their beliefs and attitudes. The objective therefore is to know the impact of stigmatizing processes on health professionals. For this reason, a systematic review of the studies available in the specialized literature is proposed. The results point to the existence of certain levels of stigma among health professionals. The opinions of the professionals towards the patients do not differ significantly from those of the general population in social distance. Discriminatory attitudes vary depending on the conditions and the pathology of the patients, with psychosis being the most punished by stigma.
\end{abstract}

Keywords: Stigma, mental health, health professionals, discrimination.

Correspondencia: Noelia Navarro Gómez. Dpto. de Psicología, Edificio C de Humanidades. Universidad de Almería. Crtra Sacramento, s/n. CP04120 La Cañada de San Urbano (Almería, España). E-mail: nng777@ual.es 


\section{Introducción}

Pese al notable avance y desarrollo en el tratamiento de los trastornos psicológicos, el estigma asociado a los mismos es un fenómeno en continuo aumento (Babic, 2010; Pescosolido, 2013). Así, los usuarios de los servicios de salud mental deben enfrentarse además de a las consecuencias que se derivan de su trastorno, a los problemas inherentes al estigma, un estresor crónico y agudo, de consecuencias devastadoras, puesto que afectan a diferentes facetas y al nivel ocupacional de quienes lo padecen, con los consiguientes perjuicios sobre la salud (Aguilar-Parra, López-Liria, Fernández-Batanero, Padilla-Góngora y Álvarez, 2016). Entre estos efectos, encontramos en la literatura, dificultad para acceder al mundo laboral, a una vivienda, así como, por descontado, dificultad en el establecimiento de relaciones interpersonales (Pereira-Pondé, Machado-Pereira y Leal, 2009; Marwaha y Johnson, 2005).

Del mismo modo, la estigmatización tiene efectos en la evolución del trastorno: guarda una correlación negativa con la calidad de vida, y la autoestima (Link y Phelan, 2001; Markowitz, 1998), la búsqueda de tratamiento y la adherencia al mismo (Bambauer y Prigerson, 2006), la aceptación de los problemas de salud mental y el compartirlo con otras personas (Scheffer, 2003), a la par que correlaciona positivamente con los síntomas de depresión y ansiedad (Ertugrul y Ulug, 2004; Markowitz, 1998) y síntomas psicóticos, así como con sentimientos de autodesprecio (Wright, Gronfein y Owens, 2000).

En definitiva, estigma es "la situación del individuo que está descalificado de la plena aceptación social" (Goffman, 1964, p.9). Así, las personas estigmatizadas poseerían ciertas características que las diferenciarían de los demás y que son juzgadas como negativas (Goffman, 1964) a través de un rasero moral variante tanto por factores históricos como culturales. Es "un conjunto de actitudes negativas y de creencias hacia la gente etiquetada como enfermos mentales y añade que se trata de fenómeno profundamente enraizado en el trasfondo de la sociedad que no solamente se da en la comunidad sino también en los servicios de salud mental" (Barbato, 2000, p.6-7).

Si bien, el proceso de estigmatización de los trastornos mentales es un hecho extendido (Link, Phelan, Bresnahan, Stueve y Pescosolido, 1999) que posee una base común de rechazo que afectaría a todos los trastornos (Farina, 1998), existiría un cierto gradiente entre trastornos psicológicos, desde aquellos más normalizados y que cuentan con la aceptación e integración social (por ej. la depresión, la ansiedad y los trastornos del comportamiento alimentario), hasta aquellos más en consonancia con la imagen tradicional de locura (esquizofrenia y otros trastornos psicóticos incluidos bajo la etiqueta genérica de trastornos mentales graves) (Crisp, 2001; López et al., 2008; Angermeyer y Dietrich, 2006; Rüsch, Angermeyer y Corrigan, 2005; Link et al., 2004; López, Saavedra, Laviana y López, 2012). Estos trastornos suscitarían actitudes similares a las que generan las drogodependencias, la prostitución y otras conductas consideradas antisociales (Corrigan y Watson, 2002; Corrigan y Penn, 1999; Marichal y Quiles, 2000; Rüsch, et al., 2005). Todo esto desembocaría en el rechazo a establecer relaciones con el enfermo, reconociendo en algunos estudios la población general entrevistada su escasa predisposición a socializarse, trabajar o integrar en la familia a una persona diagnosticada de trastorno mental (Martin, Pescosolido y Tuch, 2000).

Por otra parte, se está comenzando a poner interés también en el efecto de los procesos estigmatizantes en los profesionales de la salud, interés que se había focalizado principalmente 
en las actitudes y manifestaciones conductuales de la población general (Chin y Balon, 2006; Hinshaw, 2009). En términos generales, se ha encontrado que no existen diferencias significativas entre profesionales y población general en lo que se refiere a las creencias, actitudes y comportamientos hacia quienes padecen un trastorno mental (Nordt, Rössler y Lauber, 2006). Se ha encontrado, por ejemplo, que los profesionales, consideran a estas personas violentas (Dixon, Roberts, Lawrie, Jones y Humphreys, 2008), que manifiestan deseo de distanciamiento social (Nordt et al., 2006) dudando incluso sobe las capacidades de este colectivo para adaptarse socialmente, así como, sobre la efectividad de los tratamientos (Paksoy-Erbaydar y Cilingiroglu, 2010). De hecho, se ha encontrado que una historia previa de paciente violento es un factor que propicia que los psiquiatras diagnostiquen esquizofrenia (Clark y Rowe, 2006). Incluso se ha encontrado que las actitudes estigmatizantes se van agudizando a medida que los profesionales avanzan en su formación (Paksoy-Erbaydar y Cilingiroglu, 2010). Dado el rol de los profesionales al proporcionar apoyo terapéutico y teniendo en cuenta que teóricamente deberían ser los que mejor comprenden a sus pacientes, emitiendo sugerencias constructivas de manejo y seguimiento, estas evidencias parecen contradictorias.

Estas actitudes discriminatorias varían en función tanto de las condiciones, como de la patología de los pacientes con esta problemática. Los trastornos que soportan más lastre son los relacionados con la psicosis o el abuso de sustancias. Las atribuciones de irresponsabilidad contribuirían a potenciar este efecto (Luty, Umoh, Sessay y Sarkhel, 2007). Así, muchos profesionales de la Salud Mental prefieren no trabajar con las personas gravemente perturbadas o muestran actitudes negativas hacia las personas con psicosis (Heresco-Levy, Ermilov, Giltsinsky, Lichtenstein y Blander 1999). Podría ocurrir que estos profesionales hayan interiorizado las ideas negativas que caracterizaron a la medicina en los dos últimos siglos (Overton y Medina, 2008), así como el desprestigio social, patente en críticas y sátiras en los medios de comunicación.

En efecto, se ha encontrado la firme creencia entre los usuarios de los servicios de Salud Mental y sus familias sobre la experimentación de mayor estigma y discriminación por parte de los profesionales de este sector que de cualquier otro de la sociedad (Wagner, Torres-González, Geidel y King, 2011), y especialmente en lo que se refiere a atención primaria y urgencias, manifestando haber sufrido actitudes de rechazo, comentarios despectivos, consejos desalentadores o poco prácticos, falta de información sobre el trastorno o tratamiento, e incluso sentirse ignoradas o culpables de su padecimiento. Los principales problemas encontrados hacen referencia a que los cuidadores consideran como mecanismos de estigma desplegar comportamientos diferenciales hacia este tipo de pacientes, considerándolos como pacientes específicos de los psiquiatras; los propios protocolos y planes de atención son estigmatizadores, puesto que obvian las necesidades reales de los usuarios en lo que a ocio y tiempo libre se refieren, siendo las actividades llevadas a cabo, propuestas de los profesionales desde sus propios intereses o acordes a los recursos e infraestructuras disponibles (Badallo-Carbajosa, Ballesteros Pérez, Bertina, Cerezo-Quintana y Magro-Sánchez, 2018). Del mismo modo, éstos sostienen percibir en ocasiones estigma hacia ellos por asociación, es decir que su posición como profesional de la Salud Mental le ha llevado a situaciones en las que ellos mismos han sufrido los efectos de la estigmatización. 


\section{Método}

\section{Procedimiento}

Se ha realizado una revisión bibliográfica en diferentes bases de datos de las cuales se han obtenido 38 artículos en los que se estudia el estigma en diferentes profesionales de la salud (principalmente profesionales de la enfermería, psiquiatría y psicología). Se introdujeron los términos "profesionales de la salud", "estigma", "profesionales de la salud y enfermedad mental" en los buscadores Psycinfo, Proquest y PubMed. Además, estos artículos fueron revisados para encontrar otros artículos relevantes que no se hubieran filtrado. Como criterios de inclusión, los artículos debían estar escritos en castellano o inglés, y versar sobre la temática investigada, siendo requisito imprescindible que los profesionales ya hubieran completado su formación y se encontrasen en activo. Se han excluido los artículos escritos en otros idiomas, los artículos de revisión, así como aquellos cuya muestra eran profesionales de la salud aún en proceso de formación. Se incluyeron los artículos publicados en el período comprendido entre 2002-2018.

\section{Resultados}

Los resultados más relevantes se resumen en la tabla que se presenta a continuación: 
Tabla 1. Resumen Estudios Profesionales de la Salud y Estigma

\begin{tabular}{|c|c|c|c|c|}
\hline Autor y año & Participantes & Metodología & Objetivos & Resultados \\
\hline $\begin{array}{l}\text { Mukherjee et } \\
\text { al., } 2002\end{array}$ & 520 médicos y estudiantes de medicina & Cuestionario & $\begin{array}{l}\text { Estudiar las actitudes de los médicos y estudiantes } \\
\text { de medicina con respecto a la enfermedad mental. }\end{array}$ & $\begin{array}{l}\text { Mayor optimismo con respecto al tratamiento en los } \\
\text { profesionales y estudiantes de medicina en comparación con } \\
\text { la población general. }\end{array}$ \\
\hline $\begin{array}{l}\text { Lauber et al., } \\
2004\end{array}$ & $\begin{array}{l}90 \text { psiquiatras y } 786 \text { miembros de la } \\
\text { población general suiza. }\end{array}$ & Cuestionario & $\begin{array}{l}\text { Evaluar las actitudes de los psiquiatras hacia la } \\
\text { psiquiatría comunitaria y hacia las personas con } \\
\text { enfermedad mental grave, y compararlas con la } \\
\text { población en general. }\end{array}$ & $\begin{array}{l}\text { Tanto los psiquiatras como la población en general presentan una } \\
\text { actitud global positiva hacia los recursos en la comunidad, } \\
\text { aunque los psiquiatras en mayor medida. } \\
\text { En cuanto a la distancia social, ambos grupos no mostraban } \\
\text { diferencias significativas }\end{array}$ \\
\hline $\begin{array}{l}\text { Kingdon et } \\
\text { al., } \\
2004\end{array}$ & $\begin{array}{l}2813 \text { miembros del colegio Real de } \\
\text { Psiquiatras de Reino Unido. }\end{array}$ & Cuestionario & $\begin{array}{l}\text { Explorar las actitudes de los profesionales de la } \\
\text { psiquiatría hacia la salud mental. }\end{array}$ & $\begin{array}{l}\text { Los participantes mostraron actitudes positivas, y, en } \\
\text { cualquier caso, mejores que las de la población general hacia } \\
\text { las personas con enfermedad mental }\end{array}$ \\
\hline $\begin{array}{l}\text { Magliano et } \\
\text { al., } 2004\end{array}$ & $\begin{array}{l}190 \text { enfermeros, } 110 \text { psiquiatras y } 709 \\
\text { familiares de pacientes con } \\
\text { enfermedad mental. }\end{array}$ & Cuestionario & $\begin{array}{l}\text { Comparar las creencias sobre la esquizofrenia en } \\
\text { profesionales de la salud y familiares de pacientes } \\
\text { con enfermedad mental. }\end{array}$ & $\begin{array}{l}\text { Los psiquiatras y los enfermeros/as consideran que las } \\
\text { personas con enfermedad mental pueden trabajar y deben ser } \\
\text { castigados en caso de actos ilegales; los familiares y los } \\
\text { enfermeros/as consideran que estas personas son } \\
\text { impredecibles. }\end{array}$ \\
\hline $\begin{array}{l}\text { Tay et al., } \\
2004\end{array}$ & $\begin{array}{l}409 \text { profesionales de la enfermería de } \\
\text { Singapur. }\end{array}$ & Cuestionario & $\begin{array}{l}\text { Conocer las opiniones de las enfermeras hacia las } \\
\text { enfermedades mentales y quienes las padecen. }\end{array}$ & $\begin{array}{l}\text { Se encontraron actitudes positivas en general, especialmente en } \\
\text { aquellas con más años de experiencia. }\end{array}$ \\
\hline $\begin{array}{l}\text { Ucok et al., } \\
2004\end{array}$ & 60 psiquiatras de Turquía & Cuestionario & $\begin{array}{l}\text { Conocer las actitudes de los psiquiatras hacia la } \\
\text { esquizofrenia }\end{array}$ & $\begin{array}{l}\text { Entre otras respuestas un alto porcentaje no visitaría a un } \\
\text { paciente con esquizofrenia y se sentiría incomodo con estas } \\
\text { personas en un evento social. }\end{array}$ \\
\hline $\begin{array}{l}\text { Ishige et al., } \\
2005\end{array}$ & $\begin{array}{l}261 \text { enfermeros/as y psiquiatras, } 83 \\
\text { enfermeros de otras áreas de la salud } \\
\text { de Japón. }\end{array}$ & $\begin{array}{ll}\text { Escalas } & \mathrm{de} \\
\text { diferencial } & \\
\text { semántico } & \end{array}$ & $\begin{array}{l}\text { Evaluar las actitudes de psiquiatras y enfermeros/as } \\
\text { y compararlas con las de otros profesionales de la } \\
\text { salud y población general. }\end{array}$ & $\begin{array}{l}\text { Los psiquiatras y enfermeros/a mostraron actitudes más } \\
\text { favorables que el resto de encuestados. }\end{array}$ \\
\hline $\begin{array}{l}\text { Nordt et al., } \\
2006\end{array}$ & $\begin{array}{l}1073 \text { profesionales de la salud mental } \\
\text { y } 1737 \text { miembros de la población } \\
\text { general de Suiza }\end{array}$ & $\begin{array}{l}\text { Entrevistas } \\
\text { telefónicas }\end{array}$ & $\begin{array}{l}\text { Conocer las actitudes hacia los trastornos de la salud } \\
\text { mental y compararlos con las actitudes que genera } \\
\text { la depresión en profesionales de la salud y } \\
\text { población general. }\end{array}$ & $\begin{array}{l}\text { Los profesionales de la salud mostraron actitudes más } \\
\text { positivas que el público general. El } 60 \% \text { de los encuestados } \\
\text { de la población general retiraría el permiso de conducir a las } \\
\text { personas con trastornos mentales y el } 29 \% \text { recomendaría el } \\
\text { aborto a estas personas. En comparación con la depresión, la } \\
\text { esquizofrenia genera peores opiniones. }\end{array}$ \\
\hline $\begin{array}{l}\text { Chin et al., } \\
2006\end{array}$ & $\begin{array}{l}38 \text { psiquiatras residentes y } 29 \text { médicos } \\
\text { residentes en otras especialidades de } \\
\text { EEUU }\end{array}$ & Cuestionario & $\begin{array}{l}\text { Comparar las actitudes hacia la depresión y la } \\
\text { esquizofrenia }\end{array}$ & Se encontraron resultados más positivos hacia la depresión. \\
\hline
\end{tabular}




\begin{tabular}{|c|c|c|c|c|}
\hline Autor y año & Participantes & Metodología & Objetivos & Resultados \\
\hline $\begin{array}{l}\text { Deans et al., } \\
2006\end{array}$ & $\begin{array}{l}65 \text { profesionales de la enfermería de } \\
\text { Australia con diagnóstico de } \\
\text { personalidad límite }\end{array}$ & Cuestionario & $\begin{array}{l}\text { Conocer la opinión de estas profesionales con este } \\
\text { diagnóstico hacia los pacientes con trastorno de } \\
\text { personalidad límite }\end{array}$ & $\begin{array}{l}\text { Porcentajes bajos de adjetivos positivos. Una amplia mayoría } \\
\text { consideraron a estos pacientes como manipuladores; los } \\
\text { porcentajes de adjetivos positivos fueron bajos }\end{array}$ \\
\hline $\begin{array}{l}\text { Adewuya et } \\
\mathrm{al}, 2007\end{array}$ & 312 médicos de Nigeria & Cuestionario & $\begin{array}{l}\text { Evaluar las actitudes de médicos hacia la } \\
\text { enfermedad mental }\end{array}$ & $\begin{array}{l}\text { Las personas con enfermedad mental fueron percibidas como } \\
\text { peligrosas y la mayoría manifestaron deseo de distancia } \\
\text { social. }\end{array}$ \\
\hline $\begin{array}{l}\text { James et al., } \\
2007\end{array}$ & $\begin{array}{l}65 \text { profesionales de la enfermería en } \\
\text { Irlanda }\end{array}$ & Cuestionario & $\begin{array}{l}\text { Conocer la opinión de estas personas hacia los } \\
\text { pacientes con trastorno bipolar }\end{array}$ & $\begin{array}{l}\text { la mayoría de los participantes considero que estas personas } \\
\text { eran dificiles de cuidar, y más difíciles de tratar que otros } \\
\text { pacientes, aunque tenían tratamiento. }\end{array}$ \\
\hline $\begin{array}{l}\text { Grausgruber } \\
\text { et al.,2007 }\end{array}$ & $\begin{array}{l}460 \text { profesionales de la salud mental } \\
\text { de Austria, } 1042 \text { participantes de la } \\
\text { población general y } 137 \text { familiares de } \\
\text { personas con enfermedad mental. }\end{array}$ & Cuestionario & $\begin{array}{l}\text { Conocer las actitudes hacia la enfermedad mental } \\
\text { de estos grupos }\end{array}$ & $\begin{array}{l}\text { Los profesionales de la salud mental mostraron actitudes más } \\
\text { positivas que la población general hacia el tratamiento de la } \\
\text { esquizofrenia y sobre su peligrosidad }\end{array}$ \\
\hline $\begin{array}{l}\text { Mavundla et } \\
\text { al., } 2007\end{array}$ & 100 enfermeros de Sudáfrica & Cuestionarios & $\begin{array}{l}\text { Determinar las actitudes hacia las personas con } \\
\text { enfermedad mental en los profesionales de la } \\
\text { enfermería }\end{array}$ & $\begin{array}{l}\text { Se encontraron actitudes negativas hacia las personas con } \\
\text { enfermedad mental. }\end{array}$ \\
\hline $\begin{array}{l}\text { Munro et al., } \\
2007\end{array}$ & $\begin{array}{l}140 \text { enfermeros/as del área de salud } \\
\text { mental en Reino Unido. }\end{array}$ & Cuestionarios & $\begin{array}{l}\text { Conocer las actitudes de estos profesionales hacia } \\
\text { los trastornos de salud mental }\end{array}$ & $\begin{array}{l}\text { Los encuestados mostraron actitudes positivas hacia las } \\
\text { personas que padecen estos trastornos. }\end{array}$ \\
\hline $\begin{array}{l}\text { Servais et al., } \\
2007\end{array}$ & 306 psicólogos de la APA & Cuestionario & $\begin{array}{l}\text { Conocer las actitudes de estos profesionales hacia } \\
\text { las personas con depresión, con trastorno límite de } \\
\text { la personas y esquizofrenia. }\end{array}$ & $\begin{array}{l}\text { La esquizofrenia y el trastorno límite suscitaron las peores } \\
\text { opiniones. Las personas con esquizofrenia fueron señaladas } \\
\text { como indeseables por un } 34 \% \text { y peligrosas por el } 12 \% \text {. Las } \\
\text { personas con trastorno bipolar fueron señaladas como las más } \\
\text { peligrosas }(42 \%) \text {, la depresión suscitó mejores opiniones. }\end{array}$ \\
\hline $\begin{array}{l}\text { Bjorkman, et } \\
\text { al., } 2008\end{array}$ & $\begin{array}{l}51 \text { enfermeros de un psiquiátrico y } 69 \\
\text { de una clínica somática }\end{array}$ & Cuestionario & $\begin{array}{l}\text { Conocer las actitudes hacia la enfermedad mental } \\
\text { de estos profesionales }\end{array}$ & $\begin{array}{l}\text { Los profesionales psiquiátricos mostraron actitudes más } \\
\text { positivas, especialmente en sus reacciones hacia los pacientes } \\
\text { con esquizofrenia o con problemas de drogadicción. }\end{array}$ \\
\hline \multirow[t]{2}{*}{$\begin{array}{l}\text { Des Courtis, } \\
\text { et al., } 2008\end{array}$} & $\begin{array}{l}350 \text { psiquiatras, psicólogos, } \\
\text { enfermeros y otros terapeutas del sur } \\
\text { de Brasil }\end{array}$ & Cuestionario & $\begin{array}{l}\text { Conocer las actitudes hacia la enfermedad mental } \\
\text { de estos grupos }\end{array}$ & $\begin{array}{l}\text { Los profesionales de la salud mental mostraron opiniones } \\
\text { más positivas acerca de la integración de las personas con } \\
\text { trastornos mentales. }\end{array}$ \\
\hline & & & & $\begin{array}{l}\text { Los resultados se compararon con otros estudios } \\
\text { internacionales encontrándose que los profesionales de Brasil } \\
\text { mostraban actitudes más positivas y menor deseo de distancia } \\
\text { social. }\end{array}$ \\
\hline
\end{tabular}




\begin{tabular}{|c|c|c|c|c|}
\hline Autor y año & Participantes & Metodología & Objetivos & Resultados \\
\hline $\begin{array}{l}\text { Peris et al., } \\
2008\end{array}$ & $\begin{array}{l}5204 \text { estudiantes de medicina, } 112 \\
\text { miembros de la población general, } 541 \\
\text { trabajadores de los servicios sociales, } \\
407 \text { profesionales de la salud y } 275 \\
\text { psicólogos clínicos y graduados en } \\
\text { psicología. }\end{array}$ & $\begin{array}{l}\text { Cuestionarios y } \\
\text { medidas } \\
\text { implícitas (IAT) }\end{array}$ & $\begin{array}{l}\text { Conocer las actitudes hacia la enfermedad mental } \\
\text { de estos grupos y determinar si existen o no } \\
\text { diferencias }\end{array}$ & $\begin{array}{l}\text { El grupo de salud mental mostró actitudes más positivas tanto } \\
\text { en las medidas implícitas como explicitas que el público } \\
\text { general hacia los trastornos psicológicos. }\end{array}$ \\
\hline $\begin{array}{l}\text { Arvaniti et al, } \\
2009\end{array}$ & $\begin{array}{l}361 \text { profesionales de la salud y } 231 \\
\text { estudiantes en Grecia }\end{array}$ & $\begin{array}{l}\text { Cuestionarios y } \\
\text { escalas }\end{array}$ & $\begin{array}{l}\text { Conocer la opinión de estos profesionales y } \\
\text { estudiantes hacia la salud mental }\end{array}$ & $\begin{array}{l}\text { Una importante parte de los encuestados mostraron actitudes } \\
\text { negativas hacia las personas con enfermedad mental, } \\
\text { especialmente en lo referido a la discriminación social y la } \\
\text { restricción del paciente. }\end{array}$ \\
\hline $\begin{array}{l}\text { Chikaodiri, } \\
2009 .\end{array}$ & $\begin{array}{l}362 \text { profesionales de la salud del } \\
\text { hospital Aminu Kano de Nigeria }\end{array}$ & Cuestionario & $\begin{array}{l}\text { Conocer la opinión de los profesionales de la salud } \\
\text { haca los pacientes psiquiátricos y la enfermedad } \\
\text { mental. }\end{array}$ & $\begin{array}{l}\text { Los profesionales manifestaron miedo hacia estas personas y } \\
\text { deseo de segregación. }\end{array}$ \\
\hline $\begin{array}{l}\text { Fernando et } \\
\text { al., } 2010\end{array}$ & $\begin{array}{l}574 \text { estudiantes de medicina y } 74 \\
\text { médicos de Sri Lanka }\end{array}$ & Cuestionario & $\begin{array}{l}\text { Comparar las actitudes hacia la salud mental de } \\
\text { médicos y estudiantes de Sri Lanka con las } \\
\text { obtenidos en otros estudios internacionales }\end{array}$ & $\begin{array}{l}\text { El estudio reveló mayores niveles de estigma hacia pacientes } \\
\text { con depresión, alcohol y drogadicción en la muestra de Sri } \\
\text { Lanka en comparación con la muestra de Reino Unido, pero } \\
\text { las actitudes hacia la esquizofrenia fueron menos } \\
\text { estigmatizantes. }\end{array}$ \\
\hline $\begin{array}{l}\text { Pande et al., } \\
2011\end{array}$ & 222 profesionales de la salud. & $\begin{array}{l}\text { Cuestionario } \\
\text { semiestructurado }\end{array}$ & $\begin{array}{l}\text { Examinar las actitudes de los profesionales de la } \\
\text { salud acerca de la enfermedad mental. }\end{array}$ & $\begin{array}{l}\text { Los profesionales de la salud manifestaron el deseo de una } \\
\text { mayor formación en salud mental }\end{array}$ \\
\hline $\begin{array}{l}\text { Minas et al., } \\
2011\end{array}$ & $\begin{array}{l}\text { Dos grupos de profesionales de la salud } \\
\text { de Malasia a los que se les aplicaron } \\
\text { aleatoriamente cuestionarios sobre la } \\
\text { diabetes (298) o los trastornos mentales } \\
\text { (356). }\end{array}$ & Cuestionarios & $\begin{array}{l}\text { Examinar las actitudes de los profesionales de la } \\
\text { salud hacia la enfermedad mental en comparación } \\
\text { con la diabetes. }\end{array}$ & $\begin{array}{l}\text { Los pacientes con enfermedad mental reciben un tratamiento } \\
\text { diferencial al que reciben las personas con diabetes debido al } \\
\text { estigma. }\end{array}$ \\
\hline $\begin{array}{l}\text { Verhaeghe. et } \\
\text { al.,2012 }\end{array}$ & $\begin{array}{l}543 \text { profesionales de la salud y } 707 \\
\text { usuarios de los servicios de salud } \\
\text { mental. }\end{array}$ & Cuestionarios & $\begin{array}{l}\text { Investigar la relación entre el estigma, el burnout y } \\
\text { la satisfacción laboral en profesionales y la relación } \\
\text { entre el estigma percibido, el autoestigma y } \\
\text { satisfacción con los servicios médicos recibidos. }\end{array}$ & $\begin{array}{l}\text { El estigma en los profesionales de la salud se relaciona con la } \\
\text { despersonalización y con menos satisfacción laboral en los } \\
\text { profesionales; a mayor estigma percibido por los usuarios, } \\
\text { mayor autoestigma y menos satisfacción con los servicios de } \\
\text { salud mental recibidos. }\end{array}$ \\
\hline $\begin{array}{l}\text { Druetta et al., } \\
2013\end{array}$ & 517 profesionales de la salud mental. & Entrevistas & $\begin{array}{l}\text { Determinar las actitudes y la distancia social del os } \\
\text { profesionales de la salud hacia las personas con } \\
\text { esquizofrenia }\end{array}$ & $\begin{array}{l}\text { Los profesionales de la salud mostraron actitudes } \\
\text { estigmatizantes y deseo de distancia social hacia las personas } \\
\text { con esquizofrenia }\end{array}$ \\
\hline $\begin{array}{l}\text { Reavley, } \\
2014\end{array}$ & $\begin{array}{l}518 \text { profesionales de la salud, } 506 \\
\text { psiquiatras y } 498 \text { psicólogos clínicos y } \\
6019 \text { miembros de la población } \\
\text { general. }\end{array}$ & $\begin{array}{l}\text { Encuestas } \\
\text { encuestas } \\
\text { telefónicas }\end{array}$ & $\begin{array}{l}\text { Explorar las actitudes hacia las personas con } \\
\text { trastorno mental en los profesionales de la salud y } \\
\text { compararlas con las de la población general en } \\
\text { Australia. }\end{array}$ & $\begin{array}{l}\text { Las actitudes de la población general fueron comparables a } \\
\text { las de los profesionales de la salud en distancia social y en } \\
\text { actitudes hacia el estigma, aunque menos estigmatizantes en } \\
\text { la atribución de debilidad y peligrosidad. }\end{array}$ \\
\hline
\end{tabular}




\begin{tabular}{|c|c|c|c|c|}
\hline Autor y año & Participantes & Metodología & Objetivos & Resultados \\
\hline $\begin{array}{l}\text { Kopera et al., } \\
2015\end{array}$ & $\begin{array}{l}28 \text { estudiantes de medicina (sin } \\
\text { contacto previo) y } 29 \text { psiquiatras y } \\
\text { psicoterapeutas con al menos } 2 \text { años de } \\
\text { experiencia. }\end{array}$ & $\begin{array}{l}\text { IAT; } \\
\text { autoinforme }\end{array}$ & $\begin{array}{l}\text { Determinar las actitudes hacia las personas con } \\
\text { trastorno mental determinando la influencia del } \\
\text { contacto previo y la experiencia con estas personas. }\end{array}$ & $\begin{array}{l}\text { El contacto con las personas con trastorno mental no } \\
\text { modifica necesariamente las actitudes negativas implícitas. }\end{array}$ \\
\hline $\begin{array}{l}\text { Morgan et } \\
\text { al.,2016 }\end{array}$ & $\begin{array}{l}1381 \text { usuarios de los servicios de salud } \\
\text { mental. }\end{array}$ & $\begin{array}{l}\text { Entrevistas; } \\
\text { estudio } \\
\text { descriptivo }\end{array}$ & $\begin{array}{l}\text { Investigar los efectos de la discriminación y el } \\
\text { tratamiento positivo en adultos con problemas de } \\
\text { salud mental en Australia. }\end{array}$ & $\begin{array}{l}\text { Sólo el } 1.8 \% \text { de los participantes señalaron discriminación por } \\
\text { parte de los profesionales. El } 40.4 \% \text { señaló un trato positivo. } \\
\text { La discriminación interfirió con la recuperación. }\end{array}$ \\
\hline $\begin{array}{l}\text { Winkler et } \\
\text { al., } 2016\end{array}$ & $\begin{array}{l}1810 \text { miembros de la población } \\
\text { general y } 120 \text { médicos. }\end{array}$ & Cuestionarios & $\begin{array}{l}\text { Comparar las actitudes hacia las personas con } \\
\text { enfermedad mental entre los profesionales de la } \\
\text { salud y la población general en República Checa. }\end{array}$ & $\begin{array}{l}\text { Menor estigma en los profesionales de la salud que en la } \\
\text { población general. }\end{array}$ \\
\hline $\begin{array}{l}\text { Bannatyne et } \\
\text { al.,2017 }\end{array}$ & $\begin{array}{lllll}41 & \text { médicos y } & 85 & \text { estudiantes } & \text { de } \\
\text { psicología. } & & & \end{array}$ & Cuestionarios & $\begin{array}{l}\text { Examinar el estigma hacia la anorexia nerviosa en } \\
\text { estudiantes y profesionales de la salud mental. }\end{array}$ & $\begin{array}{l}\text { Se encontró más estigma hacia la anorexia nerviosa en los } \\
\text { hombres (atribuyeron más responsabilidad y culpa y menor } \\
\text { atribuciones biogenéticas). Los estudiantes de medicina } \\
\text { mostraron más reacciones negativas. }\end{array}$ \\
\hline $\begin{array}{l}\text { Siqueira et } \\
\text { al.,2017 }\end{array}$ & $\begin{array}{l}246 \text { profesionales de la salud de Río de } \\
\text { Janeiro. }\end{array}$ & $\begin{array}{l}\text { Estudio } \\
\text { transversal; } \\
\text { cuestionario }\end{array}$ & $\begin{array}{l}\text { Evaluar las actitudes de los profesionales de la } \\
\text { salud y comprarlos con los resultados de otras } \\
\text { partes del mundo }\end{array}$ & $\begin{array}{l}\text { En general las actitudes de los profesionales de la salud hacia } \\
\text { los usuarios de los servicios de la salud mental fueron } \\
\text { positivas. }\end{array}$ \\
\hline $\begin{array}{l}\text { Waugh et al., } \\
2017\end{array}$ & $\begin{array}{l}24 \text { profesionales de la salud ( } 13 \text { de la } \\
\text { salud mental, } 11 \text { de otras áreas). }\end{array}$ & $\begin{array}{l}\text { Entrevista } \\
\text { semiestructurada, } \\
\text { estudio } \\
\text { cualitativo }\end{array}$ & $\begin{array}{l}\text { Explorar las experiencias de estigma de los } \\
\text { profesionales de la salud mental }\end{array}$ & $\begin{array}{l}\text { Los profesionales de la salud mental mostraron miedo de } \\
\text { sufrir estigma por parte de sus colegas. Las actitudes de los } \\
\text { profesionales de la salud hacia sus colegas con trastorno } \\
\text { mental fueron positivas. }\end{array}$ \\
\hline $\begin{array}{l}\text { Ordan et al., } \\
2018\end{array}$ & 61 enfermeros/as postparto. & $\begin{array}{l}\text { Cuestionarios y } \\
\text { análisis } \\
\text { cualitativo }\end{array}$ & $\begin{array}{l}\text { Examinar el estigma en profesionales de la salud } \\
\text { hacia mujeres con trastorno mental }\end{array}$ & $\begin{array}{l}\text { Se encontraron evidencias de estigma: las mujeres con } \\
\text { trastorno mental y sus hijos recibieron menos atenciones } \\
\text { postparto }\end{array}$ \\
\hline $\begin{array}{l}\text { Cremonini et } \\
\text { al..2 } 2018\end{array}$ & 120 profesionales de la salud mental. & $\begin{array}{l}\text { Estudio } \\
\text { observacional; } \\
\text { cuestionarios }\end{array}$ & $\begin{array}{l}\text { Explorar las actitudes hacia la salud mental de los } \\
\text { profesionales }\end{array}$ & $\begin{array}{l}\text { Todos los profesionales de la salud mostraron sensiblidad y } \\
\text { actitudes positivas hacia la salud mental }\end{array}$ \\
\hline $\begin{array}{l}\text { Del Olmo- } \\
\text { Romero et al., } \\
2018\end{array}$ & $\begin{array}{l}4478 \text { profesionales de la salud de } \\
\text { España, Portugal e Italia. }\end{array}$ & Cuestionarios & $\begin{array}{l}\text { Describir y comparar las actitudes hacia la } \\
\text { enfermedad mental en profesionales de la salud de } \\
3 \text { países }\end{array}$ & $\begin{array}{l}\text { Los profesionales de la salud mental mostraron actitudes } \\
\text { positivas hacia la enfermedad mental, particularmente los } \\
\text { psicólogos y los trabajadores sociales. }\end{array}$ \\
\hline $\begin{array}{l}\text { Rose et al., } \\
2018\end{array}$ & $\begin{array}{l}374 \text { trabajadores de } \text { ONG } y \\
\text { profesionales de la salud de Australia. }\end{array}$ & Cuestionarios & $\begin{array}{l}\text { Comparar las actitudes de los trabajadores de } \\
\text { ONGs con las de profesionales de la salud mental. }\end{array}$ & $\begin{array}{l}\text { Los trabajadores de organizaciones no gubernamentales } \\
\text { mostraron actitudes más positivas que los psiquiatras, } \\
\text { psicólogos y otros profesionales de la salud. }\end{array}$ \\
\hline
\end{tabular}




\section{Discusión}

La revisión sistemática realizada nos ha permitido establecer interesantes conclusiones en lo referido al estigma en los profesionales de los servicios de salud mental hacia quienes hacen uso de estos servicios. Tras aplicar las restricciones de los criterios de exclusión finalmente se ha trabajado con un total de 38 artículos. Pese a la relevancia del tópico y el papel crucial de los profesionales en la recuperación e integración, consideramos que el fenómeno está infrarrepresentado en la literatura, aunque parece que los últimos años se está invirtiendo esta tendencia.

En primer lugar, es necesario señalar, la existencia en diversos estudios encontrados de creencias negativas en los profesionales de la salud, o, en cualquier caso, que las opiniones de estos no se desvían significativamente de las de la población general. Este fenómeno ya se ha encontrado en estudios previos con profesionales de la salud y profesionales de la salud en formación (Magliano et al.,2012; Mårtensson, Jacobsson y Engström, 2014; Horsfall, Cleary y Hunt, 2010; Fresán et al., 2012).

En efecto, se ha teorizado que, en el caso de los profesionales, el contacto prolongado con los pacientes les hace subestimar las capacidades de los mismos, capacidades a su vez que se ven mermadas por los efectos secundario del tratamiento (Paksoy y Cilingiroglu, 2009). Del mismo modo, los propios afectados por la psicopatología denuncian que el trastorno mental monopoliza sus visitas a los servicios médicos de atención primaria, obviando cualquier otra dolencia existente, en un fenómeno de eclipsamiento. Esto es, los síntomas físicos se atribuirían de forma errónea al trastorno mental (Magliano et al, 2012).

Asimismo, este contacto a largo plazo neutralizaría los efectos positivos que el contacto como estrategia de intervención antiestigma ha mostrado (Corrigan y Penn, 1999), mostrando los profesionales actitudes negativas hacia las personas con psicosis o prefiriendo no trabajar con este tipo de población (Heresco-Levy et al., 1999). Se ha encontrado también cierto efecto de deformación profesional, especialmente en los profesionales de la psiquiatría, mostrando estos más estereotipos negativos y actitudes estigmatizantes que el resto de profesionales de la salud (Jorm, Korten, Jacomb, Christensen y Henderson, 1999; Nordt, et al., 2006), o incluso actitudes en las que se obvia la parte humana, y los pacientes pasan a ser un simple objetivo de trabajo. Ejemplo de todo esto serían las conductas paternalistas, en las que se tiende a la infantilización y sobreprotección, proyectando escasas esperanzas en la recuperación (Angell, Cooke y Kovac, 2005). Por el contrario, y como resultado halagüeño, Muñoz et al. (2009), aunque también hallaron de forma frecuente actitudes de compasión y disposición a la coacción para el seguimiento del tratamiento, así como el uso de etiquetas y un lenguaje estigmatizador, encontraron resultados más favorables, en lo que a actitudes se refiere, no encontrándose tampoco estigma en lo referente a la peligrosidad y responsabilidad (de hecho el $95 \%$ de los psiquiatras presupone una peligrosidad menor a estos pacientes que el resto de la población).

La mayor parte de los estudios presentados se han focalizado en la esquizofrenia, como trastorno objeto de estudio por antonomasia. Tradicionalmente la esquizofrenia es el trastorno más en consonancia con la imagen tradicional de locura, entre otras razones por la imagen sesgada proyectada por los medios de comunicación que la asocian frecuentemente con la violencia o por las características de la sintomatología, entre otras razones. 
Los estudios disponibles proceden de países tan heterogéneos como, Nigeria, Grecia, Australia, Suiza, Japón, Irlanda, Austria, Polonia o Brasil, lo que podría indicar que pese a las posibles diferencias culturales y de filosofía de trabajo, el estigma es un fenómeno universal, aunque, sería necesario ampliar la muestra para poder establecer conclusiones en los diferentes países. Es importante tener en consideración también que las diferencias de formación entre grupos de profesionales, podría explicar las diferencias encontradas en las actitudes entre grupos (Magliano et al., 2004; Nordt et al., 2006). Parece confirmarse la existencia de diferencias entre psicólogos, psiquiatras, enfermeros y otros cuidadores, aunque no pueden establecerse generalizaciones, dado el pequeño tamaño muestral. En cuanto a categoría profesional, la mayor parte de los estudios se han centrado en los enfermeros y paradójicamente, los psiquiatras y psicólogos cuentan con una menor representación en los estudios.

Siendo este el panorama actual, sería conveniente desarrollar propuestas de intervención que compensaran esta situación, tenida cuenta el papel vital de los profesionales en la rehabilitación de los pacientes de salud mental.

\section{Referencias}

Adewuya, A. y Oguntade, A. (2007). Doctors' attitude towards people with mental illness in Western Nigeria. Social Psychiatry and Psychiatric Epidemiology, 42, 931-936.

Aguilar-Parra, J.M., López-Liria, R., Fernández-Batanero, J.M., Padilla-Góngora, D. y Álvarez, J. (2016). Influencia de la ocupación en actividades de la vida diaria y de ocio sobre la salud real de la persona mayor. Psychology, Society and Education, 8, 135-147.

Angell, B., Cooke, A., y Kovac, K. (2005). First person accounts of stigma. En P. W. Corrigan (ed.), On the stigma of mental illness: Practical strategies for research and social change (pp. 6998). Washington D.C.: Asociación Americana de Psicología.

Angermeyer, M. y Dietrich, S. (2006). Public beliefs about and actitudes towards people with mental illness: a review of population studies. Acta Psychiatrica Scandinavica, 113, 163-179.

Aruna, G., Mittal, S., Yadiyal, M.B., Acharya, C., Acharya, S. y Uppulari, C. (2016). Perception, knowledge, and attitude toward mental disorders and psychiatry among medical undergraduates in Karnataka: A cross-sectional study.Indian Journal of Psychiatry, 58(1),70-76.

Arvaniti, A., Samakouri, M., Kalamara, E., Bochtsou, V., Bikos, C. y Livaditis, M. (2009). Health service staff's attitudes towards patients with mental illness. Social Psychiatry and Psychiatric Epidemiology, 44, 658-665.

Babic, D. (2010). Stigma and mental illness. Materia Socio Médica, 22, 43-46.

Badallo-Carbajosa, A., Ballesteros-Pérez, F., Bertina, A., Cerezo-Quintana, Y. y Magro Sánchez, M. B. (2018). La dinámica estigmatizante: generación y mantenimiento del estigma y el autoestigma asociado al trastorno mental en la vida cotidiana. Clínica Contemporánea, 9, e1. https://doi.org/10.5093/cc2018a3.

Bambauer, K.Z. y Prigerson, H.G. (2006). The Stigma Receptivity Scale and its association with mental health service use among bereaved older adults. Journal of Nervous and Mental Disease, 94, 139-141.

Bannatyne. A. J. y Stapleton, P.B. (2017). Attitudes towards anorexia nervosa: volitional stigma differences in a sample of pre-clinical medicine and psychology students. Journal of Mental Health, 26(5),442-448.

Barbato, A. (2000). Consequences of schizophrenia: A public health perspective. World Association for Psychosocial Rehabilitation Bulletin, 21,6-7.

(c) Psy, Soc, \& Educ, 2019, Vol. 11(2) 
Bell, J.S., Aaltonen, S.E., Airaksinen, M.S., Volmer, D., Gharat, M.S., Muceniece, R., Vitola, A., Foulon, V., Desplenter. F.A. y Chen, T.F. (2010). Determinants of mental health stigma among pharmacy students in Australia, Belgium, Estonia, Finland, India and Latvia. International Journal of Social Psychiatry, 56(1), 3-14.

Bjorkman, T., Angelman, T. y Jonsson, M. (2008). Attitudes towards people with Mental Illness: A cross-sectional study among nursing staff in psychiatric care and somatic care. Scandinavian Journal of Caring Sciences, 22, 170-177.

Chikaodiri, A. N. (2009). Attitude of health workers to the care of psychiatric patients. Annals of General Psychiatry, 8, 19.

Chin, S. H. y Balon, R. (2006). Attitudes and perceptions toward depression and schizophrenia among residents in different medical specialties. Academic Psychiatry, 30(3), 262-263.

Clark, T. y Rowe, R. (2006). Violence, Stigma and Psychiatric Diagnosis: the Effects of a History of Violence on Psychiatric Diagnosis. Psychiatric Bulletin, 30, 254-256.

Corrigan, P. y Penn, D. L. (1999). Lessons from Social Psychology on discrediting psychiatric stigma. American Psychology, 54, 765-776.

Cremonini, V., Pagnucci, N., Giacometti, F. y Rubbi, I. (2018). Health care professionals attitudes towards mental illness: observational study performed at a public health facility in northern Italy. Archives of Psychiatric Nursing, 32(1), 24-30.

Crisp, A. (2001). The tendency to stigmatise. British Journal of Psychiatry, 178, 197-199.

Deans, C. y Meocivic, E. (2006). Attitudes of registered psychiatric nurses towards patients diagnosed with borderline personality disorder. Contemporary Nurse, 21, 43-49.

Del Olmo-Romero, F., González-Blanco, M., Sarró, S., Grácio, J., Martín-Carrasco, M., Martinez-Cabezón, A.C., Perna, G., Pomarol-Clotet, E., Varandas P., Ballesteros-Rodríguez, J., Rebolleda-Gil, C., Vanni, G., González-Fraile, E. y INTERNOS group. (2018). Mental health professionals' attitudes towards mental illness: professional and cultural factors in the INTERNOS study. European Archives of Psychiatry and Clinical Neuroscience, 1, 1-15.

Des Courtis, N., Lauber, C., Costa, C.T. y Cattapan-Ludewig, K. (2008). Beliefs about the mentally ill: a comparative study between healthcare professionals in Brazil and in Switzerland. International Review of Psychiatry, 20(6), 503-509.

Dixon, R. P., Roberts, L. M., Lawrie, S., Jones, L. A., \& Humphreys, M. S. (2008). Medical students' attitudes to psychiatric illness in primary care. Medical Education, 42(11), 1080-1087.

Druetta, I., Ceresa, M.F. y Leiderman, E.A. (2013). Attitudes of mental health workers toward people with schizophrenia. Vertex, 24(110),245-252.

Ertugrul, A. y Ulug, B. (2004). Perception of stigma among patients with schizophrenia. Social Psychiatry and Psychiatric Epidemiology, 39, 73-77.

Farina, A. (1998). Stigma. En K.T. Mueser y N. Tarrier (eds.), Handbook of social functioning in schizophrenia (pp. 247- 279). Boston: Allyn \& Bacon.

Fernando, S. M., Deane, F. P. y McLeod, H. J. (2010). Sri Lankan doctors' and medical undergraduates' attitudes towards mental illness. Social Psychiatry and Psychiatric Epidemiology, 45, 733-739.

Goffman, E. (1964). Estigma. La identidad deteriorada. Argentina: Amorrortu Editores.

Grausgruber, A., Meise, U., Katschnig, H., Schöny, W. y Fleischhacker, W.W. (2007). Patterns of social distance towards people suffering from schizophrenia in Austria: a comparison between the general public, relatives and mental health staff. Acta Psychiatrica Scandinavica, 115(4),310-319.

Heresco-Levy, U., Ermilov, M., Giltsinsky, B., Liechtenstein, M. y Blander, D. (1999). Treatmentresistant schizophrenia and staff rejection. Schizophrenia Bulletin, 25, 457-465.

Hinshaw, S.P. (2009). The mark of shame: stigma of mental illness and an agenda for change. Oxford: Oxford University Press. 
Ishige, N. y Hayashi, N. (2005). Occupation and social experience: Factors influencing attitude towards people with schizophrenia. Psychiatry and Clinical Neurosciences,59(1),89-95.

James, P.D. y Cowman, S. (2007). Psychiatric nurses' knowledge, experience, and attitudes towards clients with borderline personality disorder. Journal of Psychiatric and Mental Health Nursing, 14, 670-678.

James, B.O., Omoaregba, J. O. y Okogbenin, E.O. (2012). Stigmatising attitudes towards persons with mental illness: a survey of medical students and interns from southern Nigeria. Mental illness, 30, 4(1):e8.

Kingdon, D., Sharma, T., Hart, D. y The Schizophrenia Subgroup of The Royal College of Psychiatrists' Changing Minds Campaign. (2004). What attitudes do psychiatrists hold towards people with mental illness? Psychiatric Bulletin, 28(11), 401-406.

Kopera, M., Suszek, H., Bonar, E., Myszka, M., Gmaj, B., Ilgen, M. y Wojnar M. (2015). Evaluating explicit and implicit stigma of mental illness in mental health professionals and medical students. Community Mental Health Journal, 51(5),628-634.

Lauber, C., Anthony, M., Ajdacic-Gross, V. y Rössler, W. (2004). What about psychiatrists' attitude to mentally ill people? European Psychiatry, 19(7),423-427.

Lauber, C., Nordt, C., Braunscheig, C. y Rossler, W. (2006). Do mental health professionals stigmatize their patients? Acta Psychiatrica Scandanavia, 113, 51-59.

Law, G.U., Rostill-Brookes, H.y Goodman, D. (2009).Public stigma in health and non-healthcare students: attributions, emotions and willingness to help with adolescent self-harm. International Journal of Nursing Studies, 46(1),107-118.

Link, B. G. y Phelan, J. C. (2001). Conceptualizing Stigma. Annual Review of Sociology, 27, 363-385. http://dx.doi.org/10.1146/annurev.soc.27.1.363.

Link, B.G., Phelan, J.C., BreSnahan, M., Stueve, A. y Pescosolido, B.A. (1999). Public conceptions of mental illness: labels, causes, dengerousness, and social distance. American Journal of Public Health, 89, 1328-1333.

López, M., Laviana M., Fernández, L., López, A., Rodríguez, A. M. y Aparicio, A. (2008). La lucha contra el estigma y la discriminación en salud mental: Una estrategia compleja basada en la información disponible. Revista de la Asociación Española de Neuropsiquiatría, 101, 43-83.

López, M., Saavedra, F.J. Laviana, M. y López, M. (2012). Imágenes de la "locura", la "enfermedad mental" y la "depresión" en la ciudad de Sevilla. Psychology, Society and Education, 4, 151-168.

Luty, J., Umoh, O., Sessay, M. y Sarkhel, A. (2007). Effectiveness of Changing Minds campaign factsheets in reducing stigmatised attitudes towards mental illness. Psychiatric Bulletin, 31, 377-381.

Magliano, L., De Rosa, C., Fiorillo, A., Malangone, C., Guarneri, M., Marasco, C., Maj, M. y Working Group of the Italian National Study.(2004). Beliefs of psychiatric nurses about schizophrenia: a comparison with patients' relatives and psychiatrists. International Journal of Social Psychiatry, 50(4), 319-330.

Magliano, L., Read, J., Patalano, M., Sagliocchi, Oliviero, N., D’Ambrosio, A. y Cerrato, F. (2012) Contrarrestar el estigma hacia las personas con esquizofrenia en el ámbito sanitario: una experiencia piloto en una muestra de estudiantes italianos de medicina. Psychology, Society \& Education, 4, 169-181.

Marichal, F. y Quiles, M. (2000). La organización del estigma en categorías. Actualización de la taxonomía de Goffman. Psicothema, 12, (3), 458-465.

Markowitz, F. E. (1998). The effects of stigma on the psychological well-being and life satisfaction of persons with mental illness. Journal of Health and Social Behavior, 39, 335-347.

Martin, J. K., Pescosolido, B. A. y Tuch, S. A. (2000). Of fear and loathing: The role of disturbing behavior, labels, and causal attributions in shaping public attitudes toward people with mental illness. Journal of Health and Social Behavior, 41, 208-223. 
Marwaha, S. y Johnson, S. (2005). Views and experiencies of employment among people with psychosis: A qualitative descriptives study. International Journal of Social Psychiatry, 51, 302-316.

Mavundla, T. y Uys, L. (1997). The attitudes of nurses towards mentally ill people in a general hospital setting in Durban. Curationis, 20, 3-7.

Minas, H., Zamzam, R., Midin, M. y Cohen, A. (2011). Attitudes of Malaysian general hospital staff towards patients with mental illness and diabetes. BMC Public Health, 11, 317.

Morgan, A.J., Reavley, N.J., Jorm, A.F. y Beatson, R. (2016). Experiences of discrimination and positive treatment from health professionals: A national survey of adults with mental health problems. Australian and New Zealand Journal of Psychiatry, 50(8),754-762.

Mukherjee, R., Fialho, A., Wijetunge, A., Checinski, K. y Surgenor, T. (2002).The stigmatisation of psychiatric illness: the attitudes of medical students and doctors in a London teaching hospital. Psychiatric Bulletin, 26, 178-181.

Munro, S. y Baker, J.A. (2007). Surveying the attitudes of acute mental health nurses. Journal of Psychiatric and Mental Health Nursing 14(2),196-202.

Nordt, C., Rossler, W. y Lauber, C. (2006). Attitudes of mental health professionals toward people with schizophrenia and major depression. Schizophrenia Bulletin, 32, 709-714.

Ordan, R., Shor, R., Liebergall-Wischnitzer, M., Noble, L. y Noble, A. (2018). Nurses' professional stigma and attitudes towards postpartum women with severe mental illness. (2018). Journal of Clinical Nursing, 27, 1543-1551.

Overton, S. L. y Medina, S. L. (2008). The Stigma of Mental Illness. Journal of Counseling \& Development, 86,143-151.

Pande, V., Saini, R. y Chaudhury, S. (2011). Attitude toward mental illness amongst urban nonpsychiatric health professionals. Indian Journal of Psychiatry, 20(1), 17-20.

Pereira-Pondé, M., Machado-Pereira, C.T. y Leal, B. (2009). The role of leisure in the lives of psychotic patients: a qualitative study. Transcultural Psychiatry, 46, 328.

Peris, T.S., Teachman, B.A. y Nosek, B.A. (2008). Implicit and explicit stigma of mental illness. Journal of Nervous and Mental Disease, 196(10), 752-760.

Pescosolido, B. (2013). The Public Stigma of Mental Illness: What Do We Think; What Do We Know; What Can We Prove? Journal of Health and Social Behavior, 54, (1), 1-21.

Popescu, C. A., Buzoianu, A. D., Suciu, S. M. y Armean, S. M. (2017). Attitudes toward mentally ill patients: a comparison between Romanian and international medical students. Clujul Medical, 90(4), 401-406.

Poreddi, V., Thimmaiah, R. y Math, S. B. (2015). Attitudes toward people with mental illness among medical students. Journal of Neuroscience in rural practice, 6(3), 349-354.

Reavley, N. J., Mackinnon, A. J., Morgan, A. J. y Jorm, A. F. (2014). Stigmatising attitudes towards people with mental disorders: a comparison of Australian health professionals with the general community. Australian \& New Zealand Journal of Psychiatry, 48(5),433-441.

Rose, G., von Hippel, C., Brener, L. y von Hippel, B. (2018). Attitudes of people working in mental health non-governmental organisations in Australia: A comparison with other mental health professionals. Health Psychology, 5(1).

Rüsch, N., Angermeyer, M. y Corrigan, P. (2005). Mental illness stigma: concepts, consequences and initiatives to reduce stigma. European Psychiatry, 20, 529-539.

Scheffer, R. (2003, May). Addressing stigma: Increasing public understanding of mental illness. The standing senate committee on social affairs, science and technology. (Michael Kirby). Symposium conducted at the meeting of the standing senate committee on social affairs, science and technology, Canadá.

Sercu, C. y Bracke, P. (2016). Stigma as a structural power in mental health care reform: an ethnographic study among mental health care professionals in Belgium. Archives of Psychiatric Nursing 30, 710-716. 
Servais, L.M. y Saunders, S.M. (2007). Clinical psychologists' perceptions of persons with mental illness. Professional Psychology: Research and Practice, 38, 214-219.

Siqueira, S.R., Abelha, L., Lovisi, G.M., Sarução, K.R. y Yang, L. (2017). Attitudes Towards the Mentally Ill: A Study with Health Workers at a University Hospital in Rio de Janeiro. Psychiatric Quarterly,88(1), 25-38.

Tay, S.E., Pariyasami, S., Ravindran, K., Ali, MI. y Rowsudeen, M.T. (2004). Nurses' attitudes toward people with mental illnesses in a psychiatric hospital in Singapore. Journal of Psychosocial Nursing and Mental Health Services, 42(10), 40-47.

Totic, S., Stojiljkovic, D., Pavlovic, Z., Zaric, N., Zarkovic, B., Malic, L., Mihaljevic, M., JasovicGasic, M. y Maric, N.P. (2012). Stigmatization of 'psychiatric label' by medical and nonmedical students. International Journal of Social Psychiatry, 58(5), 455-462.

Ucok, A., Polat, A., Sartorius, N., Erkoc, S. y Atakli, C. (2004). Attitudes of psychiatrists toward patients with schizophrenia. Psychiatry and Clinical Neurosciences, 58, 89-91.

Verhaeghe. M. y Bracke, P. (2012). Associative Stigma among mental health professionals: implications for professional and service user well-being. Journal of Health and Social Behavior, 53, 17-32.

Vibha, P., Saddichha, S. y Kumar, R. (2008). Attitudes of ward attendants towards mental illness: comparisons and predictors. International Journal of Social Psychiatry, 54(5),469-478.

Wagner, L.C, Torres-González, F., Geidel, A.R. y King, M.B. (2011). Cuestiones existenciales en la esquizofrenia: percepción de portadores y cuidadores. Rev. Saúde Pública,45, 2, 401-408.

Waugh, W., Lethem, C., Sherring, S. y Henderson, C. (2017). Exploring experiences of and attitudes towards mental illness and disclosure amongst health care professionals: a qualitative study. Journal of Mental Health, 26(5), 457-463.

Winkler, P., Mladá, K., Janoušková, M., Weissová, A., Tušková, E., Csémy, L. y Evans-Lacko, S. (2016). Attitudes towards the people with mental illness: comparison between Czech medical doctors and general population. Social Psychiatry and Psychiatric Epidemiology, 51(9),12651273. 